\section{From bench to bedside and back}

\section{Cell Cycle}

editor-in-chief Mikhail V. Blagosklonny

Landes Bioscience. 6/yr. \$80 (individual), $\$ 250$ (institutional); free in 2002

Cancer Biology and Therapy

editor-in-chief Wafik S. El-Deiry

Landes Bioscience. 6/yr. \$80 (individual), $\$ 250$ (institutional); free in 2002

\section{Michelle D. Garrett}

Finally! A journal dedicated solely to my favourite topic - the cell-division cycle and what a discerning editorial board to boot. For someone working in this field, Cell Cycle was a joy to read, and for newcomers it will be invaluable in getting them up to speed on an ever-expanding area of research. But is it really necessary to have a whole journal dedicated to one particular cellular activity?

Actually the cell cycle is such a key cellular process that I am surprised that such a journal has not been developed before now. Besides, the cell cycle has clear applications to many other areas of basic biological research, including development, differentiation, cell death, senescence and disease. Cancer in particular often involves genetic alteration of one or more of the cell-cycle regulators. Cell Cycle therefore provides a forum for researchers in these areas to present new findings that involve the cell cycle, so it is a truly interdisciplinary journal of interest to a large readership.

The format of Cell Cycle is simple: there is an 'Editor's Corner', 'Views and Commentaries', 'Reviews' with a focus and finally a section presenting 'Experimental Papers'. The Editor's Corner provides a space where current issues can be highlighted, perhaps to do with a particular scientific topic, such as the interaction between basic cell-cycle research and cancer biology. It is also used to profile individuals, both past and present, who have made key contributions to research on the cell cycle and related areas. This is a valuable use of editorial space, as it reminds us of the history of this evergrowing field and of the individuals who have played a part in it.

Views and Commentaries contains reviews and comments on selected papers from the Experimental Papers section. This is a particularly useful exercise because these short articles provide background information that may not have been in the experimental paper. They are written by experts in the field who provide insight on the paper they are reviewing. Like many other journals, Cell Cycle publishes conceptual review articles. The added benefit here is that often all the reviews in an issue cover different aspects of one particular area of research in the cell-cycle field, such as mitosis, proteolysis and the cell cycle, giving the reader an overall view of the area.

The presentation of Experimental Papers has the added advantage of providing a forum where new methods can be reported. This is particularly relevant to cell-cycle research, where advances in the use of experimental techniques such as flow cytometry have been essential for the progression of the field.

As mentioned earlier, cancer is a disease in which the cell cycle is frequently misregulated. Cancer Biology and Therapy provides a comprehensive look at this and other molecular and cellular aspects of cancer biology, along with current and future cancer treatments. The readership for this journal will be broad, encompassing all who wish to learn more about cancer and current thinking on both its diagnosis and treatment. This journal will be of interest to the clinician on the front line of cancer treatment and also to the bench scientist at the forefront of biological research into this disease - it brings together basic cancer biology and the clinical aspects of this disease.

The layout of the journal follows the generic pattern of editorial comments, major reviews, original research papers and book reviews, but has several delightful nuances. The first of these, the 'Bedside to Bench' report, in many ways epitomizes the journal, as it has a very translational feel, highlighting a specific clinical area and then discussing the basic aspects of molecular and cellular biology that it may involve. Each of these articles opens with case reports on particular patients, an excellent reminder to of all of us of why we are involved in cancer research and treatment in the first place. The Bedside to Bench concept is, in many ways, the opposite of other journals, which often provide reports that go from the bench to the bedside, and I found it refreshing.

The original research papers presented in this journal are at the forefront of both basic biological research and the treatment of cancer. The added nuance here is that many are accompanied by a commentary that provides both a critique of the research paper and valuable background information that the reader may not be aware of. The commentary acts as a 'journal club' on the paper - very useful indeed, especially if you are a newcomer to that particular area.

Another unique feature is the 'Research Philosophy' section, which covers ethics and thinking on science, along with its practice. This is a topic that deserves its own section and which in other journals is often absent or relegated to occasional discussion. Cancer Biology and Therapy deserves a round of applause for highlighting an extremely important aspect of scientific research that in my opinion should be read by both newcomers to the field and the old hands.

To conclude, Cancer Biology and Therapy provides an excellent forum for the presentation of new information on the basic biology, diagnosis and treatment of cancer, the second most common cause of death worldwide. Cell Cycle, on the other hand, is a more specialist journal, but should be essential reading for anyone who has even a remote interest in the cell cycle, from undergraduate student to head of department. Both of these journals are currently only available online, and access is free at the moment.

Michelle D. Garrett is at the Cancer Research UK Centre for Cancer Therapeutics, Institute of Cancer Research, Haddow Labs, 15 Cotswold Road, Sutton, Surrey SM2 5NG, UK.

www.landesbioscience.com/journals/cellcycle. html

www.landesbioscience.com/journals/cancer.html

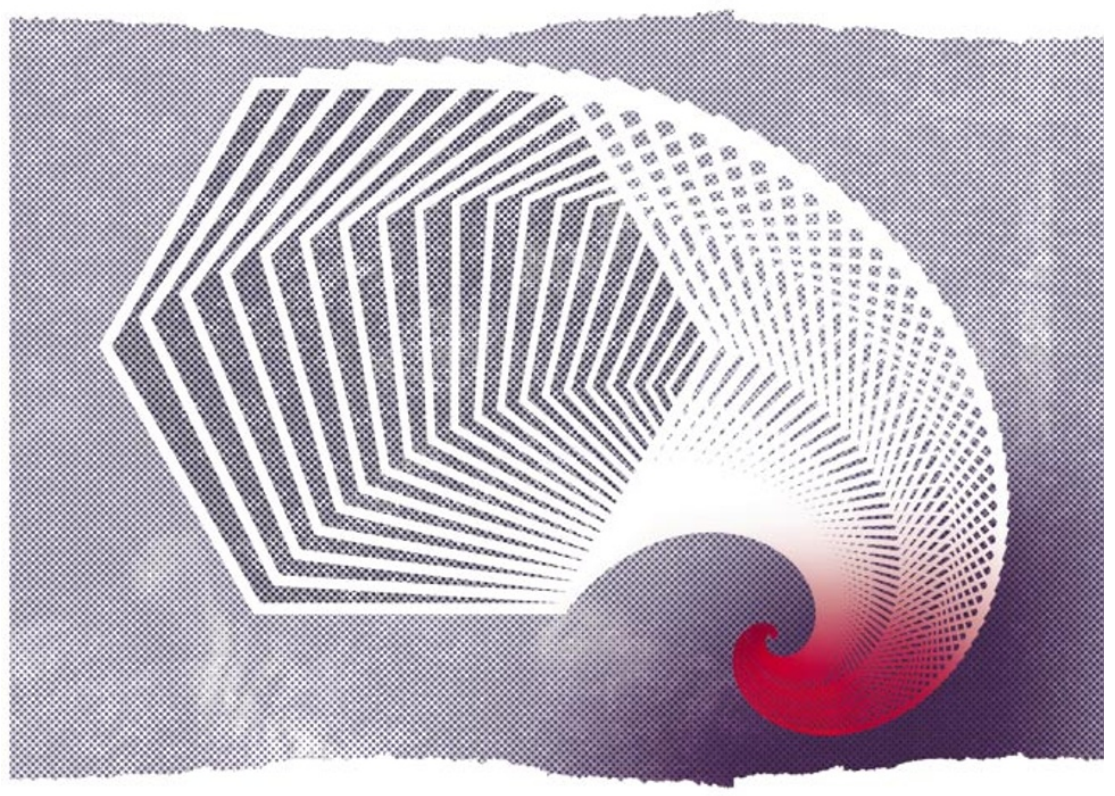

